\title{
Research methods of bioclimatic comfort at the residential area
}

\author{
Julia Gribach ${ }^{1, *}$, Olga Poddaeva ${ }^{1}$, and Pavel Churin ${ }^{1}$ \\ ${ }^{1}$ Moscow State University of Civil Engineering, Yaroslavskoe shosse, 26, Moscow, 129337, Russia
}

\begin{abstract}
One of the aspects that affect the level of environmental quality in residential development is the wind regime of the territory. To achieve the goal of ensuring bioclimatic comfort, it is necessary to carefully study the distribution of air flow at the design stage. The article presents a description and comparison of the main methods of experimental visualization of wind exposure in building conditions. The work describes in more detail the method of tracer visualization, which is used in NRU MGSU. The article presents a description of the equipment used, the basic requirements for conducting a pilot study and a description of the results obtained. The article also presents approbation of this method on the example of a projected residential complex in the city of Moscow. The paper reflects the main results obtained in this particular study, and the developed recommendations are given as an example. According to the results of the work, conclusions are presented.
\end{abstract}

\section{Introduction}

With the modern level of technology and the presence of significant scientific advances, creating the most comfortable environment for people's vital activity takes one of the leading positions in the design process [1,2]. Specialists in the construction industry are obliged to consider a significant number of factors that have a direct or indirect effect on humans or the environment, which include insolation, radiation exposure, etc. However, an important role in the creation of urban areas with high requirements is played by the main parameter of which is air flow [2,3].

Architectural objects, elements of improvement and landscaping can change the speed and direction of the wind, which affects the degree of comfort when staying on the territory of a person [4,5]. In this regard, it is necessary to scrutinize the issues of the distribution of air masses in the conditions formed at the early stages of the development of project documentation.

\section{Research methods of bioclimatic comfort}

The study of the movement of wind flows is possible by 3 methods: a method of numerical simulation, experimentally, and by combining the two methods $[5,6]$. Of course, the use of

\footnotetext{
* Corresponding author: Js-995@mail.ru
} 
computational research provides a detailed picture of the distribution of air masses, close to reality. But, on the other hand, to perform this kind of work, highly qualified specialists and the availability of powerful equipment are required, while the data obtained will be the result of a competent program setup, and, at the slightest mistake, the results will be presented with distortion

Also, the study of wind behavior in urban areas is possible using an experimental method of visualizing the flow of a gas stream [3,4,5,7]. The peculiarity of this method is the possibility of obtaining fixed patterns of airflow distribution, which allows you to visually analyze the area for the presence of uncomfortable for people zones with elevated wind speeds (over $5 \mathrm{~m} / \mathrm{s}$ ) and zones with low wind speeds (less than $1 \mathrm{~m} / \mathrm{s}$ ).

Air flow visualization occupies one of the most significant places in the study of fluid dynamics $[5,8,9]$. This is due to a greater extent with the possibility of obtaining the most qualitative and quantitative detailed information about the flow field, which can be represented in cartograms. Today, there are a sufficient number of methods and methods for obtaining visual data of air currents using optical devices, each of which provides the necessary information to solve specific tasks [10].

For example, the tinted streams method $[10,11]$ is used to visualize streamlines. The essence of this method lies in the fact that when conducting experimental studies, trickles of tinted smoke are introduced into the gas stream, which shows the trajectory of the flow. Fixation of pictures is possible with the help of photo and video, but a significant drawback is the consideration of a particular stream, while neighbouring streams will be distributed differently in the context of a specific task.

The method of filaments allows obtaining a flux spectrum directly near the object under study: bundles of filaments are attached to the surface of the body under study, which allow determining the pattern of gas flow around the body due to the change of the filament direction [11]. Photographing allows you to identify breakdowns in the flow of communication with the geometry of the body in question, as well as to identify areas with low wind speeds.

In aerodynamics, the droplet method is widely used, which consists in applying a special fluid to the body surface, which is eroded by the air flow $[11,12]$. However, the application of this visualization method in the study of the distribution of air masses in the building area (on the model of the object under study) seems to be labor-intensive. The method of the calin coating can also be referred to such (in this case, the body is completely soaked with a special liquid).

The steam screen method has found wide application in the study of the movement of air masses directly under the conditions of development [13]. In this method, the light generated in the light plane is scattered by various impurities in the stream. The light plane intersects the flow perpendicular to the velocity vector or in another direction chosen. Due to the dispersion of the luminous flux by particles, a picture of the distribution of the flow is formed, which is recorded on the optical technique. It is this method that allows to obtain information about the spatial formation of the flow.

A similar method, which will also allow to obtain patterns of distribution of wind flows in space when using laser systems, is the method of tracer visualization, which can be attributed to the class of "contactless" methods of measuring speed in flows $[14,15]$.

This research method allows recording instantaneous spatial velocity distributions, which is based on which it is possible to study flows with large-scale vortex structures.

Measurement of air velocity is based on data on the speed of movement of particles of impurities. Tracers, particles whose size is determined by minimizing the effects associated with the two-phase flow, are introduced into the air flow. The plane that is "cut" by the light beam is called the measuring flow area. The selection of this plane is based on where the movement of the particles is most visible (fig.1). 

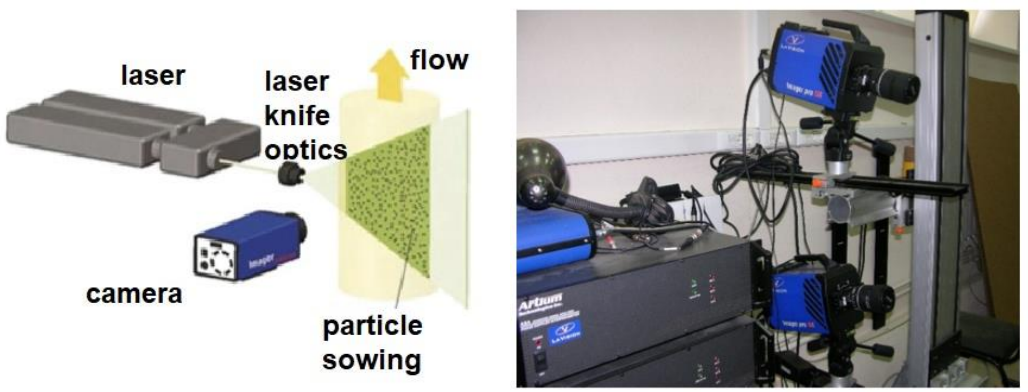

Fig. 1. The method of tracer visualization.

The analysis of the obtained data allows to obtain two-component velocity fields. The vectors obtained are projections of real velocity vectors. To perform the most accurate measurements, two recording modules are used, the orientation of the optical axes is performed at a certain angle relative to each other.

The radiation source is pulsed lasers that have a solid body[16]. Their distinctive features: short pulse duration and high energy. When using simultaneously two lasers that operate on the same optical axis, it is possible to obtain a short delay between pulses.

\section{Testing the method of tracer visualization}

Experts from the National Research Moscow State University Of Civil Engineering on the basis of the Educational, Scientific and Production Laboratory for aerodynamic and aeroacoustic testing of building structures are engaged in the study of the distribution of air flows according to this methodology. To organize the process of visualization of the wind impact on the territory of urban development, they consistently perform a series of actions on climate analysis of the studied territory, in parallel with which a physical model of the development is developed and manufactured. According to the results of the preparatory work, an experimental study of wind exposure in a wind tunnel is carried out, on the basis of which the staff of the scientific laboratory obtain a visualized picture of the distribution of air flow in the urban area. Analyzing the data, you can get the following information: pictures of weighted average fields of wind speeds with the subsequent identification of places, taking into account the distribution over the seasons.

The process of research is presented below. The object of the study is a group of residential buildings of different heights: from $30 \mathrm{~m}$ to $100 \mathrm{~m}$ (fig.2). During the work, a climatic analysis was performed, on the basis of which the most unfavorable wind directions, their speed and frequency of repetition were determined.

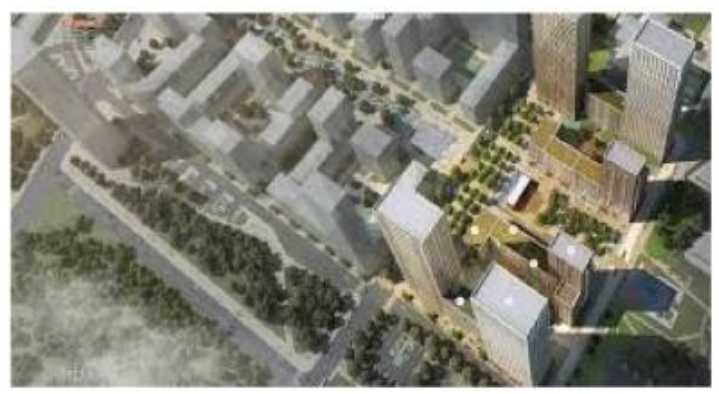

Fig. 2. The object of the study. 
Next, a reduced object model was developed and manufactured. The model is made of plexiglas $5 \mathrm{~mm}$ thick, the details of the layout were cut on a specialized laser machine, and subsequently glued with dichloroethane. The scale of the layout was chosen to be 1: 250 of the conditions for blocking the flow. After collecting all the components, the model was painted black and installed on an automated rotary table in the working area of the wind tunnel (fig.3).

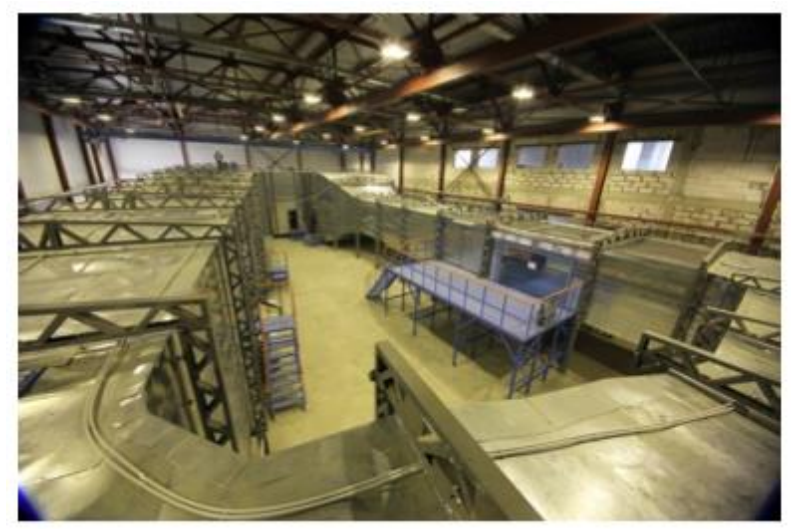

Fig. 3. The wind tunnel.

When performing a study of wind conditions in the residential area, the previously described digital tracer visualization method was used. Tracers (formula $\mathrm{C} 26 \mathrm{H} 50 \mathrm{O} 4$ ) were introduced into the airflow. The behavior of the particles in the stream was recorded on a digital high-speed camera (fig.4).

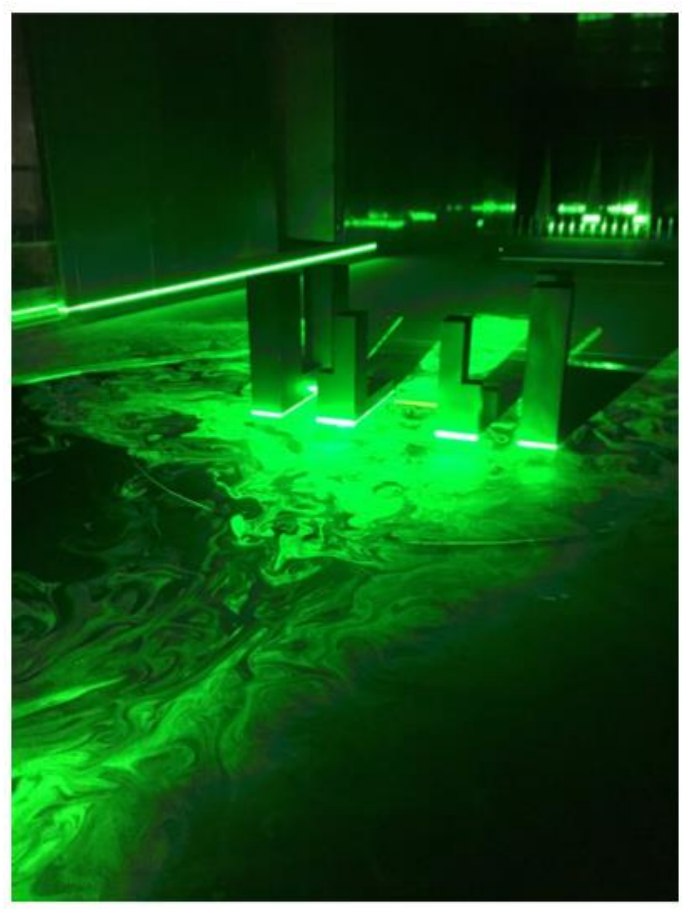

Fig.4. Conducting of the experimental research. 
As a result of the experimental studies, averaged velocity fields were obtained for 8 wind directions with a step of $45^{\circ}$, which correspond to the Moscow wind rose, after which the uncomfortable zones were identified for each air flow direction (fig.5).
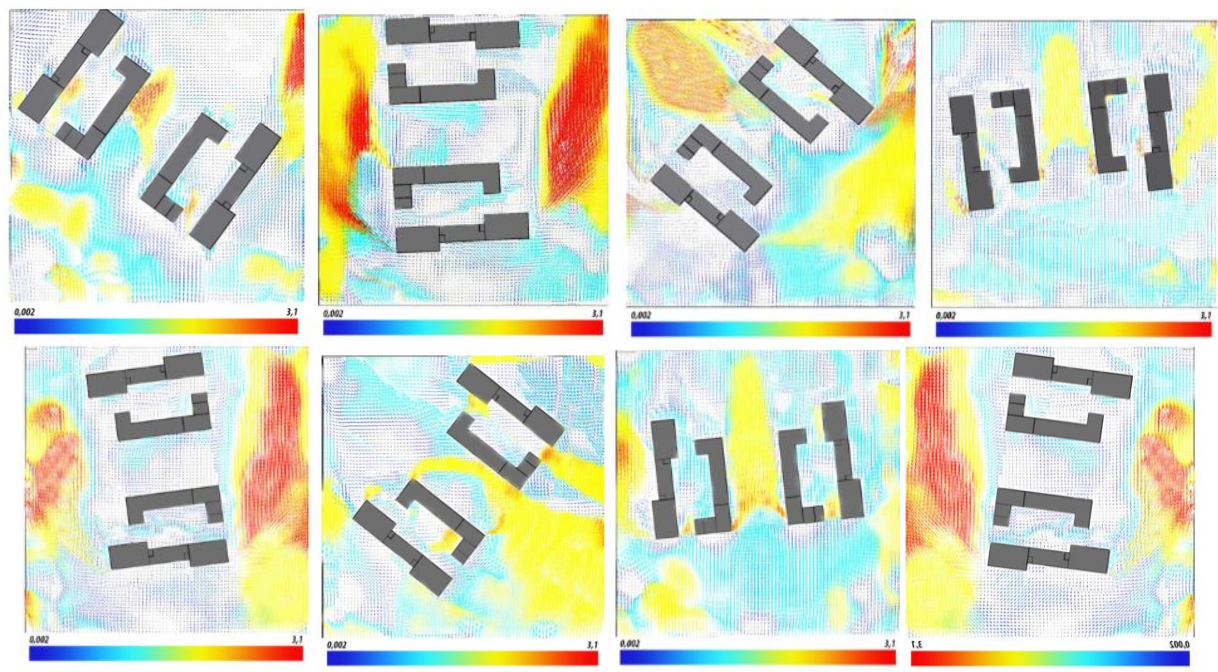

Fig. 5. Results of the experimental research.

Based on these data, specialists make recommendations for improving the quality of the environment in the residential area (fig.6).
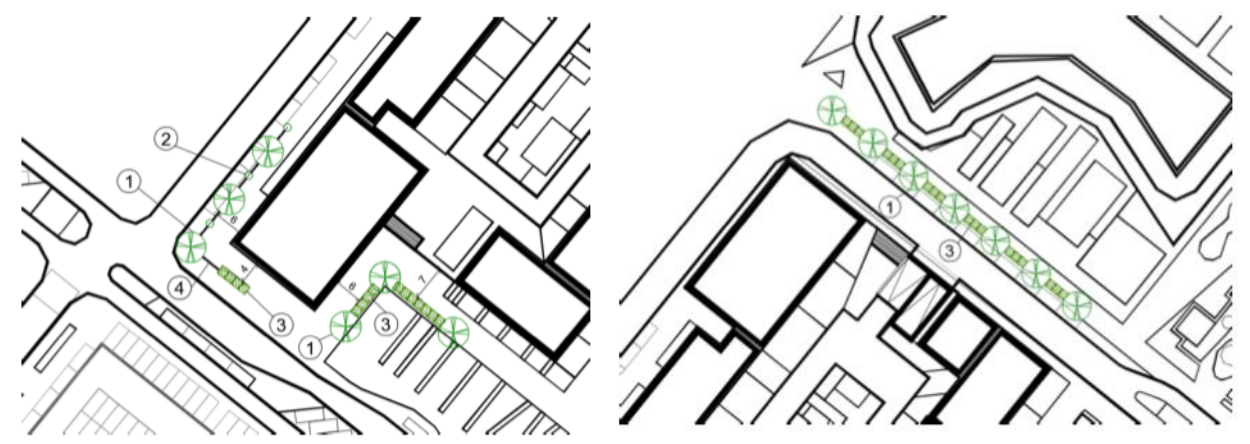

Fig. 6. Recommendations.

\section{Conclusion}

The main advantage of the method of digital tracer visualization is the possibility of obtaining instantaneous velocity fields, while other methods give us only a qualitative picture of the distribution of wind flows. In conjunction with the developed in the NRU MGSU methodology for assessing bioclimatic comfort of urban development, this method allows at the design stage to exclude the possibility of uncomfortable zones in the territory of the residential complex under study, and, if necessary, to adjust the plan of improvement of the territory taking into account aerodynamic factors at the stage of preparation of the working documentation of the project. Despite the absence of direct instructions to conduct such studies in regulatory documents, a comfortable living environment can become a significant competitive advantage in the commercial implementation of the project. 


\section{Acknowledgement}

This work was financially supported by the Ministry of Education and Science (state task $\# 7.6075 .2017 / \mathrm{Bch}$ ). All tests were carried out using research equipment of The Head Regional Shared Research Facilities and Unique Scientific Installation Large Research Gradient Wind Tunnel of the Moscow State University of Civil Engineering.

\section{References}

1. J. Alekseev, I. Dunichkin, Urban development fundamentals and reconstruction of residential buildings (Moscow, Publisher ACB, 2009)

2. J. Bezrukov, Recreational resources and balneology (Study Guide, Simferopol, 1998)

3. J. Gribach, A. Fedosova, New science: from idea to result, 172-176 (2016)

4. A. Dunichkin, O. Poddaeva, P. Churin, Evaluation of bioclimatic comfort of urban development (Moscow, Publisher NRU MGSU, 2016)

5. A. Dunichkin, O. Poddaeva, J. Gribach, IOP Conf. Series: Materials Science and Engineering 365 (2018)

6. B. Blocken, W. Janssen, T. van Hooff, Environmental Modeling and Software 30, 1534 (2012)

7. F. Serebrovsky, Construction aerodynamics and aeration of populated areas (Moscow, Publishing house on construction, 1977)

8. A. Varaksin, High Temperature 51:3, 377-407 (2013)

9. A. Bikbulatov, A. Panchenko, Bashkir Chemical Journal 20:3, 90-94 (2013)

10. A. Gebhart, Y. Galuria, R. Mahadjan, Free convective currents (1991)

11. A. Znamenskaya, L. Gvozdeva, Visualization methods in gas mechanics (Moscow, Moscow Aviation Institute, 2001)

12. A. Znamenskaya, Applied mechanics and engineering physics 36:3, 84-86 (1995)

13. V. Borovaoy, Scientific notes TsAGI 43:6, 3-17 (2012)

14. M. Litvinenko, G. Kozlov, Bulletin of the NSU 9:1, 79-86 (2014)

15. W. Woods, J. Arrington, AIAA Paper 72 (2016)

16. Whitehead, J. Hefner, AIAA Paper 77 (2016) 